\title{
Existence and Multiplicity of Nontrivial Solutions for a Class of Fourth-Order Elliptic Equations
}

\author{
Chun Li, Zeng-Qi Ou, and Chun-Lei Tang \\ School of Mathematics and Statistics, Southwest University, Chongqing 400715, China \\ Correspondence should be addressed to Chun-Lei Tang; tangcl@swu.edu.cn \\ Received 13 August 2013; Accepted 28 October 2013 \\ Academic Editor: Shaoyong Lai
}

Copyright (c) 2013 Chun Li et al. This is an open access article distributed under the Creative Commons Attribution License, which permits unrestricted use, distribution, and reproduction in any medium, provided the original work is properly cited.

Using the Fountain theorem and a version of the Local Linking theorem, we obtain some existence and multiplicity results for a class of fourth-order elliptic equations.

\section{Introduction and Main Results}

Consider the fourth-order Navier boundary value problem

$$
\begin{gathered}
\Delta^{2} u+c \Delta u+a(x) u=f(x, u), \quad \text { in } \Omega, \\
u=\Delta u=0, \quad \text { on } \partial \Omega,
\end{gathered}
$$

where $\Omega \subset \mathbb{R}^{N}(N>4)$ is a bounded smooth domain, $a \in L^{\infty}(\Omega), c \in \mathbb{R}$, and $f \in C(\Omega \times \mathbb{R}, \mathbb{R}) . \Delta$ is the Laplace operator and $\Delta^{2}$ is the biharmonic operator.

Let $0<\lambda_{1}<\lambda_{2} \leq \lambda_{3} \leq \cdots \leq \lambda_{k} \leq \cdots$ be the eigenvalues of $-\Delta$ in $H_{0}^{1}(\Omega)$. The eigenvalue problem

$$
\begin{gathered}
\Delta^{2} u+c \Delta u=\mu u, \quad \text { in } \Omega, \\
u=\Delta u=0, \quad \text { on } \partial \Omega,
\end{gathered}
$$

has infinitely many eigenvalues $\mu_{i}=\lambda_{i}\left(\lambda_{i}-c\right), i=1,2, \ldots$

We will always assume $c<\lambda_{1}$. Let $E$ be the Hilbert space $H^{2}(\Omega) \cap H_{0}^{1}(\Omega)$. $E$ is equipped with the inner product

$$
\langle u, v\rangle=\int_{\Omega}(\Delta u \Delta v-c \nabla u \nabla v) d x
$$

and the norm

$$
\|u\|=\left(\int_{\Omega}\left(|\Delta u|^{2}-c|\nabla u|^{2}\right) d x\right)^{1 / 2} .
$$

A weak solution of problem (1) is any $u \in E$ such that

$$
\int_{\Omega}(\Delta u \Delta v-c \nabla u \cdot \nabla v+a(x) u v) d x-\int_{\Omega} f(x, u) v d x=0
$$

for any $v \in E$.

Let $\varphi: E \rightarrow \mathbb{R}$ be the functional defined by

$$
\varphi(u)=\frac{1}{2} \int_{\Omega}\left(|\Delta u|^{2}-c|\nabla u|^{2}+a(x) u^{2}\right) d x-\int_{\Omega} F(x, u) d x,
$$

where $F(x, u)=\int_{0}^{u} f(x, s) d s$. And, one has

$$
\begin{aligned}
\left\langle\varphi^{\prime}(u), v\right\rangle= & \int_{\Omega}(\Delta u \Delta v-c \nabla u \cdot \nabla v+a(x) u v) d x \\
& -\int_{\Omega} f(x, u) v d x
\end{aligned}
$$

for any $u, v \in E$, so that a critical point of the functional $\varphi$ in $E$ corresponds to a weak solution of problem (1).

In recent years, fourth-order problems have been studied by many authors. In [1], Lazer and McKenna have pointed out that problem (1) furnishes a model to study travelling waves in suspension bridges if $f(x, u)=b\left((u+1)^{+}-1\right)$, where $u^{+}=$ $\max \{u, 0\}$ and $b \in \mathbb{R}$. Since then, more general nonlinear fourth-order elliptic boundary value problems have been studied. 
In $[2,3]$, Micheletti and Pistoia proved that problem

$$
\begin{gathered}
\Delta^{2} u+c \Delta u=f(x, u), \quad \text { in } \Omega, \\
u=\Delta u=0, \quad \text { on } \Omega,
\end{gathered}
$$

admits two or three solutions by variational method. In [4], Zhang obtained the existence of weak solutions for problem (8) when $f(x, u)$ is sublinear at $\infty$. In [5], Zhang and Li showed that problem (8) has at least two nontrivial solutions by means of Morse theory and local linking. When $f(x, u)$ is asymptotically linear at infinity, the existence of three nontrivial solutions has been obtained in [6] by using Morse theory. In [7], by using the mountain pass theorem, An and Liu gave the existence result for nontrivial solutions for a class of asymptotically linear fourth-order elliptic equations. In [8], Zhou and Wu got the existence of four sign-changing solutions or infinitely many sign-changing solutions for (8) by using the sign-changing critical point theorems. In [9], Yang and Zhang showed new results on invariant sets of the gradient flows of the corresponding variational functionals and proved the existence of positive, negative, and signchanging solutions for some fourth-order semilinear elliptic boundary value problems. In [10], by using the variational method, Liu and Huang obtained an existence result of signchanging solutions as well as positive and negative solutions for a fourth-order elliptic problem whose nonlinear term is asymptotically linear at both zero and infinity.

In this paper, we will study the existence of nontrivial solutions of problem (1). Our main results are the following theorems.

Theorem 1. Assume that $F$ is even in $u$ and the following conditions hold:

$\left(F_{1}\right)$

$$
\frac{F(x, u)}{u^{2}} \longrightarrow+\infty \text { as }|u| \longrightarrow \infty
$$

uniformly in $x \in \Omega$;

$\left(F_{2}\right)$ there exist two constants $2<\theta<2 N /(N-4)=2^{* *}$ and $k_{1}>0$ such that

$$
|f(x, u)| \leq k_{1}\left(1+|u|^{\theta-1}\right)
$$

uniformly for all $x \in \Omega$;

$\left(F_{3}\right)$ there exists a constant $\gamma>N(\theta-2) / 4$ such that

$$
\liminf _{|u| \rightarrow \infty} \frac{f(x, u) u-2 F(x, u)}{|u|^{\gamma}}>0,
$$

uniformly for all $x \in \Omega$.

Then problem (1) has infinitely many nontrivial solutions.

Theorem 2. Assume that $F$ satisfies $\left(F_{1}\right)$ and

$\left(F_{4}\right)$ there exist three positive constants $L, m_{1}$, and $m_{2}$ such that

$$
\begin{aligned}
& \left(j_{1}\right) f(x, u) u-2 F(x, u) \geq m_{1}|u|^{2}, \text { if }|u| \geq L ; \\
& \left(j_{2}\right)|f(x, u)|^{\sigma} /|u|^{\sigma} \leq m_{2}(f(x, u) u-2 F(x, u)), \text { if } \\
& |u| \geq L, \text { where } \sigma>(N-2) / 2 .
\end{aligned}
$$

If $F$ is even in $u$, problem (1) has infinitely many nontrivial solutions.

Remark 3. For Schrödinger equation, the corresponding condition $\left(F_{4}\right)$ is due to Ding and Luan [11]. The condition $\left(F_{4}\right)$ is weaker than the usual Ambrosetti-Rabinowitz-type condition (see $[11,12])$.

Theorem 4. Assume that $F$ satisfies $\left(F_{1}\right),\left(F_{2}\right),\left(F_{3}\right)$, and

$$
\frac{F(x, u)}{u^{2}} \longrightarrow 0 \quad \text { as } u \longrightarrow 0
$$

uniformly in $x \in \Omega$.

If 0 is an eigenvalue of $\Delta^{2}+c \Delta+a$ (with Navier boundary condition), assume also the condition that

$\left(F_{6}\right)$ there exists $\delta>0$ such that

(i) $F(x, u) \geq 0$, for all $|u| \leq \delta, x \in \Omega$; or

(ii) $F(x, u) \leq 0$, for all $|u| \leq \delta, x \in \Omega$.

Then problem (1) has at least one nontrivial solution.

Theorem 5. Suppose that $F$ satisfies $\left(F_{1}\right),\left(F_{4}\right)$, and $\left(F_{5}\right)$. If 0 is an eigenvalue of $\Delta^{2}+c \Delta+a$ (with Navier boundary condition), assuming also $\left(F_{6}\right)$, then problem (1) has at least one nontrivial solution.

Here, we have

$$
\begin{aligned}
\varphi(u) & =\frac{1}{2} \int_{\Omega}\left(|\Delta u|^{2}-c|\nabla u|^{2}+a(x) u^{2}\right) d x-\int_{\Omega} F(x, u) d x \\
& =\frac{1}{2}\left(\left\|u^{+}\right\|^{2}-\left\|u^{-}\right\|^{2}\right)-\int_{\Omega} F(x, u) d x
\end{aligned}
$$

where $u^{-} \in E^{-}, u^{+} \in E^{+}$, and $E^{-}\left(E^{+}\right)$is the space spanned by the eigenvectors corresponding to negative (positive) eigenvalues of $\Delta^{2}+c \Delta+a$. It is easy to know that $\varphi \in C^{1}(E, \mathbb{R})$ under the conditions of our theorems.

It is well known that $E$ is continuously embedded in $L^{\lambda}(\Omega)$ for every $\lambda \in[1,2 N /(N-4)]$. If $1 \leq \lambda<2 N /(N-4)$, the embedding is compact. It follows from $\left(F_{2}\right),\left(F_{3}\right)$, and $\left(F_{4}\right)$ that

$$
\theta<\frac{2 N}{N-4}, \quad \frac{\theta N-4 \gamma}{N-4}<\frac{2 N}{N-4}, \quad \frac{2 \sigma}{\sigma-1}<\frac{2 N}{N-4} .
$$

Hence, there is a positive constant $K$ such that

$$
\|u\|_{L^{\lambda}} \leq K\|u\|, \quad \forall u \in E
$$

for $\lambda=1,2, \theta, 2 \sigma /(\sigma-1), 2 N /(N-4)=2^{* *}$, where $\|\cdot\|_{L^{\lambda}}$ denotes the norm of $L^{\lambda}(\Omega)$. 


\section{Proof of Main Results}

In this paper, we will use the Fountain Theorem of Bartsch ([13, Theorem 2.5], [14, Theorem 3.6]) to prove our Theorems 1 and 2 . And, we will prove Theorems 4 and 5 by using a version of Local Linking theorem [12, Theorem 2.2] which extends theorems given by Li and Willem [15], Li and Szulkin [16].

In $[13,14]$, Bartsch established the Fountain Theorem under the $(\mathrm{PS})_{c}$ condition. Since the Deformation Theorem is still valid under the Cerami condition, the Fountain Theorem is true under the Cerami condition. So, we have the following Fountain Theorem.

Let $X$ be a reflexive and separable Banach space. It is well known that there exist $\left\{v_{n}\right\}_{n \in \mathbb{N}} \subset X,\left\{\psi_{n}\right\}_{n \in \mathbb{N}} \subset X^{*}$ such that

(1) $\left\langle\psi_{n}, v_{m}\right\rangle=\delta_{n, m}$ where $\delta_{n, m}=1$ for $n=m$ and $\delta_{n, m}=0$ for $n \neq m$.

(2) $\overline{\operatorname{span}}\left\{v_{n} n \in \mathbb{N}\right\}=X, \overline{\operatorname{span}}^{\omega^{*}}\left\{\psi_{n} \mid n \in \mathbb{N}\right\}=X^{*}$.

Let $X_{j}=\mathbb{R} v_{j}$; then $X=\overline{\bigoplus_{j \geq 1} X_{j}}$. We define

$$
Y_{k}=\bigoplus_{j=1}^{k} X_{j}, \quad Z_{k}=\overline{\bigoplus_{j=k}^{\infty} X_{j}}
$$

Theorem A (Fountain theorem). Assume that $\varphi \in C^{1}(X, \mathbb{R})$ satisfies the Cerami condition $(C), \varphi(-u)=\varphi(u)$. If for almost every $k \in \mathbb{N}$, there exist $\rho_{k}>r_{k}>0$ such that

$\left(A_{1}\right)$

$$
a_{k}:=\max _{u \in Y_{k},\|u\|=\rho_{k}} \varphi(u) \leq 0,
$$

$\left(A_{2}\right)$

$$
b_{k}:=\inf _{u \in Z_{k},\|u\|=r_{k}} \varphi(u) \longrightarrow \infty, \quad k \longrightarrow \infty,
$$

then $\varphi$ has an unbounded sequence of critical values.

For the reader's convenience, we state the following Local Linking theorem [12, Theorem 2.2]. Let $X$ be a real Banach space with $X=X^{1} \oplus X^{2}$ and $X_{0}^{j} \subset X_{1}^{j} \subset X_{2}^{j} \subset \cdots \subset X^{j}$ such that $X^{j}=\overline{\bigcup_{n \in \mathbb{N}} X_{n}^{j}}, j=1,2$. For every multi-index $\alpha=$ $\left(\alpha_{1}, \alpha_{2}\right) \in \mathbb{N}^{2}$, let $X_{\alpha}=X_{\alpha_{1}}^{1} \oplus X_{\alpha_{2}}^{2}$. We define that $\alpha \leq \beta \Leftrightarrow$ $\alpha_{1} \leq \beta_{1}, \alpha_{2} \leq \beta_{2}$. A sequence $\left\{\alpha_{n}\right\} \subset \mathbb{N}^{2}$ is admissible if for every $\alpha \in \mathbb{N}^{2}$ there is $m \in \mathbb{N}$ such that $n \geq m \Rightarrow \alpha_{n} \geq \alpha$. We say that $\varphi \in C^{1}(X, \mathbb{R})$ satisfies the $\left(C^{*}\right)$ condition if every sequence $\left\{u_{\alpha_{n}}\right\}$ such that $\left\{\alpha_{n}\right\}$ is admissible and satisfies

$$
\begin{gathered}
u_{\alpha_{n}} \in X_{\alpha_{n}}, \quad \sup _{n} \varphi\left(u_{\alpha_{n}}\right)<\infty, \\
\left(1+\left\|u_{\alpha_{n}}\right\|\right) \varphi_{\alpha_{n}}^{\prime}\left(u_{\alpha_{n}}\right) \longrightarrow 0
\end{gathered}
$$

contains a subsequence which converges to a critical point of $\varphi$, where $\varphi_{\alpha}=\left.\varphi\right|_{X_{\alpha}}$.

Theorem B (see [12, Theorem 2.2]). Suppose that $\varphi \in$ $C^{1}(X, \mathbb{R})$ satisfies the following assumptions: $\left(i_{1}\right) X \neq\{0\}$ and $\varphi$ has a local linking at 0 ; that is, for some $r>0$,

$$
\begin{aligned}
& \varphi(u) \geq 0, \quad \forall u \in X^{1} \text { with }\|u\| \leq r, \\
& \varphi(u) \leq 0, \quad \forall u \in X^{2} \text { with }\|u\| \leq r ;
\end{aligned}
$$

$\left(i_{2}\right) \varphi$ satisfies $\left(C^{*}\right)$ condition;

$\left(i_{3}\right) \varphi$ maps bounded sets into bounded sets;

$\left(i_{4}\right)$ for every $m \in \mathbb{N}, \varphi(u) \rightarrow-\infty$ as $|u| \rightarrow \infty$, on $u \in X_{m}^{1} \oplus X^{2}$.

Then $\varphi$ has at least one nonzero critical point.

Now, we can give the proof of our theorems.

Proof of Theorem 1. At first, we claim that $\varphi$ satisfies the Cerami condition $(C)$. Consider a sequence $\left\{u_{n}\right\}$ such that $\varphi\left(u_{n}\right)$ is bounded and $\left\|\varphi^{\prime}\left(u_{n}\right)\right\|\left(1+\left\|u_{n}\right\|\right) \rightarrow 0$ as $n \rightarrow \infty$. Then there exists a constant $M_{1}>0$ such that

$$
\left|\varphi\left(u_{n}\right)\right| \leq M_{1}, \quad\left(1+\left\|u_{n}\right\|\right)\left\|\varphi_{n}^{\prime}\left(u_{n}\right)\right\| \leq M_{1} .
$$

By a standard argument, we only need to prove that $\left\{u_{n}\right\}$ is a bounded sequence in $E$. Otherwise, going if necessary to a subsequence, we can assume that $\left\|u_{n}\right\| \rightarrow \infty$ as $n \rightarrow \infty$. From $\left(F_{3}\right)$, there exist two constants $k_{2}, k_{3}>0$ such that

$$
f(x, u) u-2 F(x, u) \geq k_{2}|u|^{\gamma}-k_{3}, \quad \forall(x, u) \in \Omega \times \mathbb{R} .
$$

So, by (21) and (22), we have

$$
\begin{aligned}
3 M_{1} & \geq 2 \varphi\left(u_{n}\right)-\left\langle\varphi^{\prime}\left(u_{n}\right), u_{n}\right\rangle \\
& =\int_{\Omega}\left(f\left(x, u_{n}\right) u_{n}-2 F\left(x, u_{n}\right)\right) d x \\
& \geq k_{2} \int_{\Omega}\left|u_{n}\right|^{\gamma} d x-k_{3}|\Omega|
\end{aligned}
$$

which implies that

$$
\int_{\Omega}\left|u_{n}\right|^{\gamma} d x<k_{4}
$$

for all $n \in \mathbb{N}$ and some positive constant $k_{4}$.

Since

$$
\gamma>\frac{N}{4}(\theta-2), \quad \frac{N}{4}(\theta-2)<\frac{2 N}{N+4}(\theta-1)<\frac{2 N}{N-4} \text {. }
$$

On the one hand, we consider the case

$$
\frac{N}{4}(\theta-2)<\gamma<\frac{2 N}{N+4}(\theta-1) .
$$

Putting

$$
\alpha=\frac{2(\theta-1) N-(N+4) \gamma}{2 N-(N-4) \gamma}
$$


one has $0<\alpha<1$. Let

$$
p=\frac{\gamma}{\theta-1-\alpha}>1, \quad u_{n}=u_{n}^{+}+u_{n}^{-}+u_{n}^{0} \in E^{+} \oplus E^{-} \oplus E^{0},
$$

where $E^{0}=\operatorname{ker}\left(\Delta^{2}+c \Delta+a\right)$. We can obtain from Hölder's inequality, (15), and (24) that

$$
\begin{aligned}
\int_{\Omega}\left|u_{n}\right|^{\theta-1}\left|u_{n}^{+}\right| d x & \\
= & \int_{\Omega}\left|u_{n}\right|^{\theta-1-\alpha}\left|u_{n}\right|^{\alpha}\left|u_{n}^{+}\right| d x \\
& =\int_{\Omega}\left|u_{n}\right|^{\gamma / p}\left|u_{n}\right|^{\alpha}\left|u_{n}^{+}\right| d x \\
\leq & \left(\int_{\Omega}\left(\left|u_{n}\right|^{\gamma / p}\right)^{p} d x\right)^{1 / p}\left(\int_{\Omega}\left(\left|u_{n}\right|^{\alpha}\left|u_{n}^{+}\right|\right)^{q} d x\right)^{1 / q} \\
\leq & \left(\int_{\Omega}\left|u_{n}\right|^{\gamma} d x\right)^{1 / p}\left(\int_{\Omega}\left(\left|u_{n}\right|^{q \alpha}\right)^{2^{* *} /(q \alpha)} d x\right)^{\alpha / 2^{* *}} \\
& \times\left(\int_{\Omega}\left(\left|u_{n}^{+}\right|^{q}\right)^{2^{* *} / q} d x\right)^{1 / 2^{* *}} \\
\leq & k_{4}^{1 / p}\left\|u_{n}\right\|_{L^{2^{* *}}}^{\alpha}\left\|u_{n}^{+}\right\| L_{L^{2 *}} \\
\leq & k_{4}^{1 / p} K^{\alpha+1}\left\|u_{n}\right\|^{\alpha}\left\|u_{n}^{+}\right\|
\end{aligned}
$$

for all $n$, where $q=p /(p-1)=2^{* *} /(\alpha+1)$.

By $\left(F_{2}\right)$ and (29), one has

$$
\begin{aligned}
\left\langle\varphi^{\prime}\left(u_{n}\right), u_{n}^{+}\right\rangle & =\left\|u_{n}^{+}\right\|^{2}-\int_{\Omega} f\left(x, u_{n}\right) u_{n}^{+} d x \\
& \geq\left\|u_{n}^{+}\right\|^{2}-\int_{\Omega}\left|f\left(x, u_{n}\right)\right|\left|u_{n}^{+}\right| d x \\
& \geq\left\|u_{n}^{+}\right\|^{2}-k_{1} \int_{\Omega}\left(\left|u_{n}\right|^{\theta-1}\left|u_{n}^{+}\right|+\left|u_{n}^{+}\right|\right) d x \\
& =\left\|u_{n}^{+}\right\|^{2}-k_{1} \int_{\Omega}\left|u_{n}\right|^{\theta-1}\left|u_{n}^{+}\right| d x-k_{1} \int_{\Omega}\left|u_{n}^{+}\right| d x \\
& \geq\left\|u_{n}^{+}\right\|^{2}-k_{1} k_{4}^{1 / p} K^{\alpha+1}\left\|u_{n}\right\|^{\alpha}\left\|u_{n}^{+}\right\|-k_{1} K\left\|u_{n}^{+}\right\|
\end{aligned}
$$

for all $n$. Since $\alpha<1$, we have

$$
\frac{\left\|u_{n}^{+}\right\|}{\left\|u_{n}\right\|} \longrightarrow 0, \quad \text { as } n \longrightarrow \infty .
$$

On the other hand, if $\gamma$ satisfies,

$$
\frac{2 N}{N+4}(\theta-1) \leq \gamma<\frac{2 N}{N-4} .
$$

then one sees $1 \leq \gamma /(\gamma-\theta+1) \leq 2 N /(N-4)$. So, we get

$$
\|u\|_{L^{\gamma /(\gamma-\theta+1)}} \leq K\|u\|, \quad \forall u \in E .
$$

It follows from (24) that

$$
\frac{\int_{\Omega}\left|u_{n}\right|^{\gamma} d x}{\left\|u_{n}\right\|} \longrightarrow 0, \quad \text { as } n \longrightarrow \infty \text {. }
$$

By $\left(F_{2}\right)$ and (33), we obtain

$$
\begin{aligned}
\left\langle\varphi^{\prime}\left(u_{n}\right), u_{n}^{+}\right\rangle & \\
= & \left\|u_{n}^{+}\right\|^{2}-\int_{\Omega} f\left(x, u_{n}\right) u_{n}^{+} d x \\
\geq & \left\|u_{n}^{+}\right\|^{2}-\int_{\Omega}\left|f\left(x, u_{n}\right)\right|\left|u_{n}^{+}\right| d x \\
\geq & \left\|u_{n}^{+}\right\|^{2}-k_{1} \int_{\Omega}\left(\left|u_{n}\right|^{\theta-1}\left|u_{n}^{+}\right|+\left|u_{n}^{+}\right|\right) d x \\
= & \left\|u_{n}^{+}\right\|^{2}-k_{1} \int_{\Omega}\left|u_{n}\right|^{\theta-1}\left|u_{n}^{+}\right| d x-k_{1} \int_{\Omega}\left|u_{n}^{+}\right| d x \\
\geq & \left\|u_{n}^{+}\right\|^{2}-k_{1}\left(\int_{\Omega}\left(\left|u_{n}\right|^{\theta-1}\right)^{\gamma / \theta-1} d x\right)^{(\theta-1) / \gamma} \\
& \times\left(\int_{\Omega}\left|u_{n}^{+}\right|^{\gamma /(\gamma-\theta+1)} d x\right)^{(\gamma-\theta+1) / \gamma}-k_{1} K\left\|u_{n}^{+}\right\| \\
= & \left\|u_{n}^{+}\right\|^{2}-k_{1}\left\|u_{n}\right\|_{L^{\gamma}}^{\theta-1} \cdot\left\|u_{n}^{+}\right\|_{L^{\gamma /(\gamma-\theta+1)}}-k_{1} K\left\|u_{n}^{+}\right\| \\
\geq & \left\|u_{n}^{+}\right\|^{2}-k_{1} K\left\|u_{n}\right\|_{L^{\gamma}}^{\theta-1} \cdot\left\|u_{n}^{+}\right\|-k_{1} K\left\|u_{n}^{+}\right\|
\end{aligned}
$$

for all $n$. Note that $\gamma \geq 2 N(\theta-1) /(N+4)$ and $N>4$ imply that $\gamma>\theta-1$. So, it follows from (34) and the above expression that

$$
\frac{\left\|u_{n}^{+}\right\|}{\left\|u_{n}\right\|} \longrightarrow 0, \quad \text { as } n \longrightarrow \infty \text {. }
$$

Hence, we conclude from (31) and (36) that

$$
\frac{\left\|u_{n}^{+}\right\|}{\left\|u_{n}\right\|} \longrightarrow 0, \quad \text { as } n \longrightarrow \infty \text {. }
$$

Similarly for $u_{n}^{-}$, we get

$$
\frac{\left\|u_{n}^{-}\right\|}{\left\|u_{n}\right\|} \longrightarrow 0, \quad \text { as } n \longrightarrow \infty \text {. }
$$

It follows from the equivalence of the norms on the finite dimensional space $E^{0}$ that there exists $K_{1}>0$ such that

$$
\begin{gathered}
\|u\|_{\infty} \leq K_{1}\|u\|, \quad\|u\| \leq K_{1}\|u\|_{L^{1}}, \\
\|u\| \leq K_{1}\|u\|_{L^{2}}, \quad \forall u \in E^{0} .
\end{gathered}
$$

Putting $\tau=2 N+(5-N) \gamma+1$, one has

$$
1<\frac{2 \tau-\gamma}{\tau-1}<\frac{2 N}{N-4} .
$$


It follows from (24), (39), and Hölder's inequality that

$$
\begin{aligned}
\frac{1}{K_{1}^{2}}\left\|u_{n}^{0}\right\|^{2} & \leq \int_{\Omega}\left|u_{n}^{0}\right|^{2} d x \\
& \leq \int_{\Omega}\left|u_{n}\right|^{2} d x=\int_{\Omega}\left|u_{n}\right|^{\gamma / \tau}\left|u_{n}\right|^{2-\gamma / \tau} d x \\
& \leq\left(\int_{\Omega}\left|u_{n}\right|^{\gamma} d x\right)^{1 / \tau}\left(\int_{\Omega}\left|u_{n}\right|^{(2 \tau-\gamma) /(\tau-1)} d x\right)^{(\tau-1) / \tau} \\
& \leq k_{4}^{1 / \tau} K^{(2 \tau-\gamma) / \tau}\left\|u_{n}\right\|^{(2 \tau-\gamma) / \tau}
\end{aligned}
$$

and consequently

$$
\frac{\left\|u_{n}^{0}\right\|}{\left\|u_{n}\right\|} \longrightarrow 0, \quad \text { as } n \longrightarrow \infty .
$$

Hence, by (37), (38), and (42), one sees

$$
1=\frac{\left\|u_{n}\right\|}{\left\|u_{n}\right\|} \leq \frac{\left\|u_{n}^{+}\right\|+\left\|u_{n}^{0}\right\|+\left\|u_{n}^{-}\right\|}{\left\|u_{n}\right\|} \longrightarrow 0
$$

as $n \rightarrow \infty$, which is a contradiction. So, we obtain that $\left\{u_{n}\right\}$ is bounded in $E$. By a standard argument, we get that $\varphi$ satisfies the condition $(C)$.

Let $E=\overline{\oplus_{j \geq 1} X_{j}}$ with $\operatorname{dim} X_{j}<\infty$ for any $j \geq 1$. Set

$$
Y_{k}=\bigoplus_{j=1}^{k} X_{j}, \quad Z_{k}=\overline{\bigoplus_{j=k}^{\infty} X_{j}} .
$$

Since $\operatorname{dim}\left(Y_{k}\right)<\infty$, all the norms are equivalent. For $u \in Y_{k}$, there exists a constant $K_{2}>0$ such that

$$
\|u\| \leq K_{2}\|u\|_{L^{2}}
$$

From condition $\left(F_{1}\right)$, there exists $k_{5}>0$ such that

$$
F(x, u) \geq K_{2}^{2}|u|^{2}-k_{5}, \quad \forall(x, u) \in \Omega \times \mathbb{R} .
$$

For $u \in Y_{k}$, it follows from (45) and (46) that

$$
\begin{aligned}
\varphi(u) & =\frac{1}{2}\left(\left\|u^{+}\right\|^{2}-\left\|u^{-}\right\|^{2}\right)-\int_{\Omega} F(x, u) d x \\
& \leq \frac{1}{2}\left(\left\|u^{+}\right\|^{2}-\left\|u^{-}\right\|^{2}\right)-K_{2}^{2}\|u\|_{L^{2}}^{2}+k_{5}|\Omega| \\
& \leq \frac{1}{2}\left(\left\|u^{+}\right\|^{2}-\left\|u^{-}\right\|^{2}\right)-\left(\left\|u^{+}\right\|^{2}+\left\|u^{0}\right\|^{2}\right)+k_{5}|\Omega| \\
& =-\frac{1}{2}\left\|u^{+}\right\|^{2}-\frac{1}{2}\left\|u^{-}\right\|^{2}-\left\|u^{0}\right\|^{2}+k_{5}|\Omega| \\
& \leq-\frac{1}{2}\|u\|^{2}+k_{5}|\Omega|
\end{aligned}
$$

which implies that

$$
\varphi(u) \longrightarrow-\infty \text { as }\|u\| \longrightarrow \infty, \quad \text { in } Y_{k} .
$$

So, $\left(A_{1}\right)$ of Theorem $\mathrm{A}$ is satisfied for every $\rho_{k}>0$ large enough.

Here, we obtain from $\left(F_{2}\right)$ that there exists a positive constant $k_{6}$ such that

$$
|F(x, u)| \leq k_{6}\left(1+|u|^{\theta}\right)
$$

uniformly for all $x \in \Omega$. Let us define

$$
\beta_{k}=\sup _{u \in Z_{k},\|u\|=1}\|u\|_{L^{\theta}} .
$$

For $k$ large enough, one has $Z_{k} \subset E^{+}$. By (49), on $Z_{k}$, we have

$$
\begin{aligned}
\varphi(u) & =\frac{\|u\|^{2}}{2}-\int_{\Omega} F(x, u) d x \\
& \geq \frac{\|u\|^{2}}{2}-k_{6}\|u\|_{L^{\theta}}^{\theta}-k_{6}|\Omega| \\
& \geq \frac{\|u\|^{2}}{2}-k_{6} \beta_{k}^{\theta}\|u\|^{\theta}-k_{6}|\Omega| .
\end{aligned}
$$

Choosing $r_{k}=\left(k_{6} \theta \beta_{k}^{\theta}\right)^{1 /(2-\theta)}$, we obtain, if $u \in Z_{k}$ and $\|u\|=$ $r_{k}$,

$$
\varphi(u) \geq\left(\frac{1}{2}-\frac{1}{\theta}\right)\left(k_{6} \theta \beta_{k}^{\theta}\right)^{2 /(2-\theta)}-k_{6}|\Omega|
$$

Since, by Lemma 3.8 of [14], $\beta_{k} \rightarrow 0$ as $k \rightarrow \infty,\left(A_{2}\right)$ is proved. Hence, the proof is completed by using Fountain theorem.

Proof of Theorem 2. Firstly, we claim that $\varphi$ satisfies the Cerami condition (C). Consider a sequence $\left\{u_{n}\right\}$ such that $\varphi\left(u_{n}\right)$ is bounded from above and $\left\|\varphi^{\prime}\left(u_{n}\right)\right\|\left(1+\left\|u_{n}\right\|\right) \rightarrow 0$ as $n \rightarrow \infty$. By a standard argument, we only need to prove that $\left\{u_{n}\right\}$ is a bounded sequence in $E$. For otherwise, we can assume that $\left\|u_{n}\right\| \rightarrow \infty$ as $n \rightarrow \infty$.

From assumption $\left(F_{4}\right)$, there exist two positive constants $m_{3}$ and $m_{4}$, such that

$$
\begin{aligned}
m_{3} & \geq 2 \varphi\left(u_{n}\right)-\left\langle\varphi^{\prime}\left(u_{n}\right), u_{n}\right\rangle \\
& =\int_{\Omega}\left(f\left(x, u_{n}\right) u_{n}-2 F\left(x, u_{n}\right)\right) d x \\
& \geq \int_{\Omega} m_{1}\left|u_{n}\right|^{2} d x-m_{4}|\Omega| .
\end{aligned}
$$

So, one has

$$
\int_{\Omega}\left|u_{n}\right|^{2} d x \leq m_{5}
$$

for all $n$ and some positive constant $m_{5}$

Let $v_{n}=u_{n} /\left\|u_{n}\right\|$; then $\left\|v_{n}\right\|=1$ and $\left\|v_{n}\right\|_{L^{r}} \leq C_{r}$ for all $r \in[1,2 N /(N-4))$. By (54), we have

$$
\int_{\Omega} v_{n}^{2} d x=\frac{1}{\left\|u_{n}\right\|^{2}} \int_{\Omega}\left|u_{n}\right|^{2} d x \leq \frac{m_{5}}{\left\|u_{n}\right\|^{2}} \longrightarrow 0
$$


as $n \rightarrow \infty$. So, for $r \in(2,(2 N-4) /(N-4))$, it follows from Hölder's inequality and the above expression that

$$
\int_{\Omega}\left|v_{n}\right|^{r} d x \leq\left(\int_{\Omega}\left|v_{n}\right|^{2(r-1)} d x\right)^{1 / 2}\left(\int_{\Omega}\left|v_{n}\right|^{2} d x\right)^{1 / 2} \longrightarrow 0
$$

as $n \rightarrow \infty$. It follows from (39) that

$$
\begin{aligned}
& \left\langle\varphi^{\prime}\left(u_{n}\right), u_{n}^{+}-u_{n}^{-}\right\rangle \\
& =\left\|u_{n}^{+}-u_{n}^{-}\right\|^{2}-\int_{\Omega} f\left(x, u_{n}\right)\left(u_{n}^{+}-u_{n}^{-}\right) d x \\
& =\left\|u_{n}\right\|^{2}-\left\|u_{n}^{0}\right\|^{2}-\int_{\Omega} f\left(x, u_{n}\right)\left(u_{n}^{+}-u_{n}^{-}\right) d x \\
& =\left\|u_{n}\right\|^{2}\left(1-\int_{\Omega} \frac{f\left(x, u_{n}\right)\left(u_{n}^{+}-u_{n}^{-}\right)}{\left\|u_{n}\right\|^{2}} d x\right)-\left\|u_{n}^{0}\right\|^{2} \\
& \geq\left\|u_{n}\right\|^{2}\left(1-\int_{\Omega} \frac{f\left(x, u_{n}\right)\left(u_{n}^{+}-u_{n}^{-}\right)}{\left\|u_{n}\right\|^{2}} d x\right)-K_{1}^{2}\left\|u_{n}^{0}\right\|_{L^{2}}^{2} .
\end{aligned}
$$

Hence, we get

$$
1-\int_{\Omega} \frac{f\left(x, u_{n}\right)\left(u_{n}^{+}-u_{n}^{-}\right)}{\left\|u_{n}\right\|^{2}} d x=o(1) .
$$

From $\left(F_{4}\right),(53)$, and (56), there exists a positive constant $m_{6}$ such that

$$
\begin{aligned}
\left|\int_{\Omega} \frac{f\left(x, u_{n}\right)\left(u_{n}^{+}-u_{n}^{-}\right)}{\left\|u_{n}\right\|^{2}} d x\right| & \leq 2 \int_{\Omega} \frac{\left|f\left(x, u_{n}\right)\right|}{\left|u_{n}\right|}\left|v_{n}\right|^{2} d x \\
& \leq 2\left(\int_{\Omega}\left(\frac{\left|f\left(x, u_{n}\right)\right|}{\left|u_{n}\right|}\right)^{\sigma} d x\right)^{1 / \sigma} \\
& \times\left(\int_{\Omega}\left|v_{n}\right|^{2 \sigma^{\prime}} d x\right)^{1 / \sigma^{\prime}} \\
& \leq m_{6}\left(\int_{\Omega}\left|v_{n}\right|^{2 \sigma^{\prime}} d x\right)^{1 / \sigma^{\prime}} \rightarrow 0
\end{aligned}
$$

as $n \rightarrow \infty$; therefore, $1=o(1)$, which is a contradiction. Hence, $\left\{u_{n}\right\}$ is bounded.

In a way similar to the proof of Theorem 1, we can obtain that $\varphi$ satisfies $\left(A_{1}\right)$ of Theorem $\mathrm{A}$.

It follows from $\left(F_{1}\right)$ that there is $L_{1}>0$ such that

$$
F(x, u) \geq|u|^{2}, \quad \forall|u| \geq L_{1} .
$$

Then, by $\left(F_{4}\right)$ and (60), for $|u| \geq L_{2}=\max \left\{L, L_{1}\right\}, x \in \Omega$, one has

$$
\begin{aligned}
|f(x, u)|^{\sigma} & \leq m_{2}(f(x, u) u-2 F(x, u))|u|^{\sigma} \\
& \leq m_{2}|f(x, u)||u|^{\sigma+1}
\end{aligned}
$$

which implies that

$$
|f(x, u)| \leq m_{2}^{1 /(\sigma-1)}|u|^{(\sigma+1) /(\sigma-1)}
$$

for $|u| \geq L_{2}$.

Therefore, there exist two positive constants $m_{7}$ and $m_{8}$ such that

$$
|F(x, u)| \leq m_{7}+m_{8}|u|^{2 \sigma /(\sigma-1)}, \quad \forall(x, u) \in \Omega \times \mathbb{R},
$$

where $2 \sigma /(\sigma-1)<2 N /(N-4)$. As the proof of Theorem 1 , we can get $\left(A_{2}\right)$. Therefore, Theorem 2 holds.

Proof of Theorem 4. The proof of this theorem is divided in several steps.

Step 1 . We claim that $\varphi$ has a local linking at zero with respect to $\left(X^{1}, X^{2}\right)$.

By $\left(F_{5}\right)$, for any $\varepsilon>0$, there exists $\delta_{1}>0$ such that

$$
|F(x, u)| \leq \varepsilon u^{2}, \quad \forall|u| \leq \delta_{1} .
$$

We obtain from the above expression and (49) that

$$
|F(x, u)| \leq \varepsilon u^{2}+M_{2}|u|^{\theta}, \quad \forall(x, u) \in \Omega \times \mathbb{R},
$$

where $M_{2}=k_{6}+k_{6} \delta_{1}^{-\theta}$. Hence, we can get from (15) and (65) that

$$
\begin{aligned}
\left|\int_{\Omega} F(x, u) d x\right| & \leq \int_{\Omega} \varepsilon u^{2} d x+M_{2} \int_{\Omega}|u|^{\theta} d x \\
& =\varepsilon\|u\|_{L^{2}}^{2}+M_{2}\|u\|_{L^{\theta}}^{\theta} \\
& \leq K^{2} \varepsilon\|u\|^{2}+K^{\theta} M_{2}\|u\|^{\theta}
\end{aligned}
$$

for all $u \in E$.

Here, we consider only the case where 0 is an eigenvalue of $\Delta^{2}+c \Delta+a$ and case (ii) of $\left(F_{6}\right)$ holds. The case (i) is similar.

Let $X=E, X^{1}=E^{+} \oplus E^{0}$, and $X^{2}=E^{-}$, where $E^{0}=$ $\operatorname{ker}\left(\Delta^{2}+c \Delta+a\right)$. Choose a Hilbertian basis $\left\{e_{n}\right\}_{n \geq 0}$ for $X^{1}$ and define

$$
\begin{gathered}
X_{n}^{1}=\operatorname{span}\left\{e_{0}, \ldots, e_{n}\right\}, \quad n \in \mathbb{N}, \\
X_{n}^{2}=X^{2}, \quad n \in \mathbb{N}, \\
X^{1}=\overline{\bigcup_{n} X_{n}^{1}} .
\end{gathered}
$$

Now, by (66), for each $u \in X^{2}=E^{-}$, one has

$$
\begin{aligned}
\varphi(u) & =-\frac{1}{2}\|u\|^{2}-\int_{\Omega} F(x, u) d x \\
& \leq-\frac{1}{2}\|u\|^{2}+K^{2} \varepsilon\|u\|^{2}+K^{\theta} M_{2}\|u\|^{\theta}
\end{aligned}
$$

Letting $\varepsilon=1 /\left(8 K^{2}\right)$ and by $\theta>2$, we have

$$
\varphi(u) \leq 0, \quad \forall u \in X^{2} \quad \text { with }\|u\| \leq \delta_{2}
$$

for $\delta_{2}>0$ small enough. 
Let $u=u^{0}+u^{+} \in E^{0} \oplus E^{+}=X^{1}$ be such that $\|u\| \leq \delta_{3}:=$ $\delta /\left(2 K_{1}\right)$. Put

$$
\Omega_{1}=\left\{x \in \Omega|| u^{+}(x) \mid \leq \frac{\delta}{2}\right\}, \quad \Omega_{2}=\Omega \backslash \Omega_{1} .
$$

Then, for all $\|u\| \leq \delta_{3}$ and $x \in \Omega$, by (39), one sees

$$
\left|u^{0}(x)\right| \leq\left\|u^{0}\right\|_{\infty} \leq K_{1}\left\|u^{0}\right\| \leq K_{1}\|u\| \leq \frac{\delta}{2} .
$$

On one hand, from above expression, for any $x \in \Omega_{1}$, we have

$$
|u(x)| \leq\left|u^{0}(x)\right|+\left|u^{+}(x)\right| \leq\left\|u^{0}\right\|_{\infty}+\frac{\delta}{2} \leq \delta .
$$

Hence, by condition (ii) of $\left(F_{6}\right)$, we get

$$
\int_{\Omega_{1}} F(x, u) d x \leq 0 .
$$

On the other hand, for any $x \in \Omega_{2}$, one has

$$
|u(x)| \leq\left|u^{0}(x)\right|+\left|u^{+}(x)\right| \leq \frac{\delta}{2}+\left|u^{+}(x)\right| \leq 2\left|u^{+}(x)\right| \text {. }
$$

Hence, for all $x \in \Omega_{2}$ and $u \in X^{1}$ with $\|u\| \leq \delta_{3}$, we can obtain from (65) that

$$
F(x, u) \leq \varepsilon u^{2}+M_{2}|u|^{\theta} \leq 4 \varepsilon\left|u^{+}(x)\right|^{2}+2^{\theta} M_{2}\left|u^{+}(x)\right|^{\theta},
$$

which implies that

$$
\begin{aligned}
\int_{\Omega_{2}} F(x, u) d x & \leq 4 \varepsilon \int_{\Omega_{2}}\left|u^{+}(x)\right|^{2} d x+2^{\theta} M_{2} \int_{\Omega_{2}}\left|u^{+}(x)\right|^{\theta} d x \\
& =4 \varepsilon\left\|u^{+}\right\|_{L^{2}}^{2}+2^{\theta} M_{2}\left\|u^{+}\right\|_{L^{\theta}}^{\theta} \\
& \leq 4 K^{2} \varepsilon\left\|u^{+}\right\|^{2}+(2 K)^{\theta} M_{2}\left\|u^{+}\right\|^{\theta} .
\end{aligned}
$$

Letting $\varepsilon=1 /\left(16 K^{2}\right)$ in above expression, then for all $x \in \Omega_{2}$ and $u \in X^{1}$ with $\|u\| \leq \delta_{3}$, we have

$$
\begin{aligned}
\varphi(u) & =\frac{1}{2}\left\|u^{+}\right\|^{2}-\int_{\Omega_{2}} F(x, u) d x-\int_{\Omega_{1}} F(x, u) d x \\
& \geq \frac{1}{2}\left\|u^{+}\right\|^{2}-4 K^{2} \varepsilon\left\|u^{+}\right\|^{2}-(2 K)^{\theta} M_{2}\left\|u^{+}\right\|^{\theta} \\
& \geq \frac{1}{4}\left\|u^{+}\right\|^{2}-(2 K)^{\theta} M_{2}\left\|u^{+}\right\|^{\theta},
\end{aligned}
$$

which implies that

$$
\varphi(u) \geq 0, \quad \forall u \in X^{1} \quad \text { with }\|u\| \leq \delta_{4}
$$

for $\delta_{4}>0$ small enough. Hence, $\varphi$ has a local linking at zero with respect to $\left(X^{1}, X^{2}\right)$ for $\delta_{5}=\min \left\{\delta_{2}, \delta_{4}\right\}$ small enough.

Step 2. In a way similar to the proof of Theorem 1, we can get that $\varphi$ satisfies the $\left(C^{*}\right)$ condition.

Step 3. Now, we claim that for each $m \in \mathbb{N}$, one has

$$
\varphi(u) \longrightarrow-\infty, \quad \text { as }\|u\| \longrightarrow \infty, \quad \text { in } X_{m}^{1} \oplus X^{2} .
$$

Since $\operatorname{dim}\left(E^{0}\right)<\infty$ and $\operatorname{dim}\left(X_{m}^{1}\right)<\infty$, all the norms are equivalent. For $u \in X_{m}^{1} \oplus X^{2}$, there exists a constant $k_{7}>0$ such that

$$
\|u\| \leq k_{7}\|u\|_{L^{2}}
$$

From condition $\left(F_{1}\right)$, there exists a constant $k_{8}>0$ such that

$$
F(x, u) \geq k_{7}^{2}|u|^{2}-k_{8}, \quad \forall(x, u) \in \Omega \times \mathbb{R} .
$$

For $u \in X_{m}^{1} \oplus X^{2}$, it follows from (80) and (81) that

$$
\begin{aligned}
\varphi(u) & =\frac{1}{2}\left(\left\|u^{+}\right\|^{2}-\left\|u^{-}\right\|^{2}\right)-\int_{\Omega} F(x, u) d x \\
& \leq \frac{1}{2}\left(\left\|u^{+}\right\|^{2}-\left\|u^{-}\right\|^{2}\right)-k_{7}^{2}\|u\|_{L^{2}}^{2}+k_{8}|\Omega| \\
& \leq \frac{1}{2}\left(\left\|u^{+}\right\|^{2}-\left\|u^{-}\right\|^{2}\right)-k_{7}^{2}\left(\left\|u^{+}\right\|_{L^{2}}^{2}+\left\|u^{0}\right\|_{L^{2}}^{2}\right)+k_{8}|\Omega| \\
& \leq \frac{1}{2}\left(\left\|u^{+}\right\|^{2}-\left\|u^{-}\right\|^{2}\right)-\left(\left\|u^{+}\right\|^{2}+\left\|u^{0}\right\|^{2}\right)+k_{8}|\Omega| \\
& =-\frac{1}{2}\left\|u^{+}\right\|^{2}-\frac{1}{2}\left\|u^{-}\right\|^{2}-\left\|u^{0}\right\|^{2}+k_{8}|\Omega| \\
& \leq-\frac{1}{2}\|u\|^{2}+k_{8}|\Omega|
\end{aligned}
$$

which implies that

$$
\varphi(u) \longrightarrow-\infty \quad \text { as }\|u\| \longrightarrow \infty, \quad \text { in } X_{m}^{1} \oplus X^{2} .
$$

Hence, all the assumptions of Theorem B are verified. Then, the proof of Theorem 4 is completed.

Proof of Theorem 5. In a way similar to the proof of Theorems 2 and 4 , we can obtain that $\varphi$ satisfies $\left(i_{1}\right),\left(i_{2}\right),\left(i_{3}\right)$, and $\left(i_{4}\right)$ of Theorem B. Therefore, Theorem 5 holds.

\section{Conflict of Interests}

The authors declare that there is no conflict of interests regarding the publication of this paper.

\section{Acknowledgments}

The authors would like to thank the referee for the valuable suggestions. This paper is supported by the National Natural Science Foundation of China (no. 11071198) and the Fundamental Research Funds for the Central Universities (no. XDJK2010C055).

\section{References}

[1] A. C. Lazer and P. J. McKenna, "Large-amplitude periodic oscillations in suspension bridges: some new connections with nonlinear analysis," SIAM Review, vol. 32, no. 4, pp. 537-578, 1990.

[2] A. M. Micheletti and A. Pistoia, "Multiplicity results for a fourth-order semilinear elliptic problem," Nonlinear Analysis A, vol. 31, no. 7, pp. 895-908, 1998. 
[3] A. M. Micheletti and A. Pistoia, "Nontrivial solutions for some fourth order semilinear elliptic problems," Nonlinear Analysis A, vol. 34, no. 4, pp. 509-523, 1998.

[4] J. Zhang, "Existence results for some fourth-order nonlinear elliptic problems," Nonlinear Analysis A, vol. 45, no. 1, pp. 2936, 2001.

[5] J. Zhang and S. Li, "Multiple nontrivial solutions for some fourth-order semilinear elliptic problems," Nonlinear Analysis $A$, vol. 60, no. 2, pp. 221-230, 2005.

[6] A. X. Qian and S. J. Li, "Multiple solutions for a fourthorder asymptotically linear elliptic problem," Acta Mathematica Sinica, vol. 22, no. 4, pp. 1121-1126, 2006.

[7] Y. An and R. Liu, "Existence of nontrivial solutions of an asymptotically linear fourth-order elliptic equation," Nonlinear Analysis A, vol. 68, no. 11, pp. 3325-3331, 2008.

[8] J. Zhou and X. Wu, "Sign-changing solutions for some fourthorder nonlinear elliptic problems," Journal of Mathematical Analysis and Applications, vol. 342, no. 1, pp. 542-558, 2008.

[9] Y. Yang and J. Zhang, "Existence of solutions for some fourthorder nonlinear elliptic problems," Journal of Mathematical Analysis and Applications, vol. 351, no. 1, pp. 128-137, 2009.

[10] X. Liu and Y. Huang, "On sign-changing solution for a fourthorder asymptotically linear elliptic problem," Nonlinear Analysis A, vol. 72, no. 5, pp. 2271-2276, 2010.

[11] Y. Ding and S. Luan, "Multiple solutions for a class of nonlinear Schrödinger equations," Journal of Differential Equations, vol. 207, no. 2, pp. 423-457, 2004.

[12] S. Luan and A. Mao, "Periodic solutions for a class of nonautonomous Hamiltonian systems," Nonlinear Analysis A, vol. 61, no. 8, pp. 1413-1426, 2005.

[13] T. Bartsch, "Infinitely many solutions of a symmetric Dirichlet problem," Nonlinear Analysis A, vol. 20, no. 10, pp. 1205-1216, 1993.

[14] M. Willem, Minimax Theorems, Birkhäuser Boston, Boston, Mass, USA, 1996.

[15] S. J. Li and M. Willem, "Applications of local linking to critical point theory," Journal of Mathematical Analysis and Applications, vol. 189, no. 1, pp. 6-32, 1995.

[16] S. J. Li and A. Szulkin, "Periodic solutions for a class of nonautonomous Hamiltonian systems," Journal of Differential Equations, vol. 112, no. 1, pp. 226-238, 1994. 


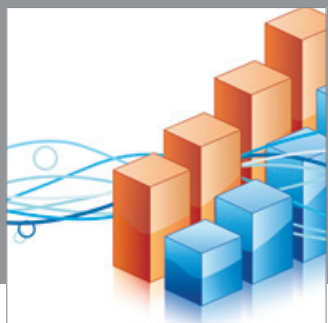

Advances in

Operations Research

mansans

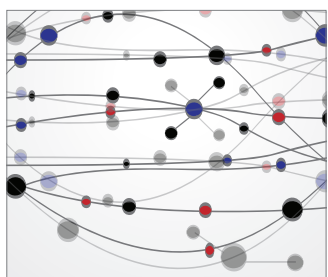

The Scientific World Journal
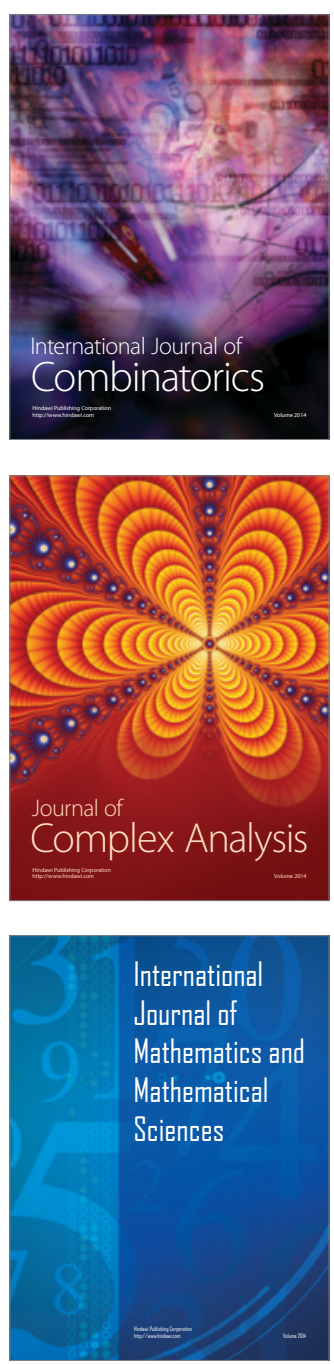
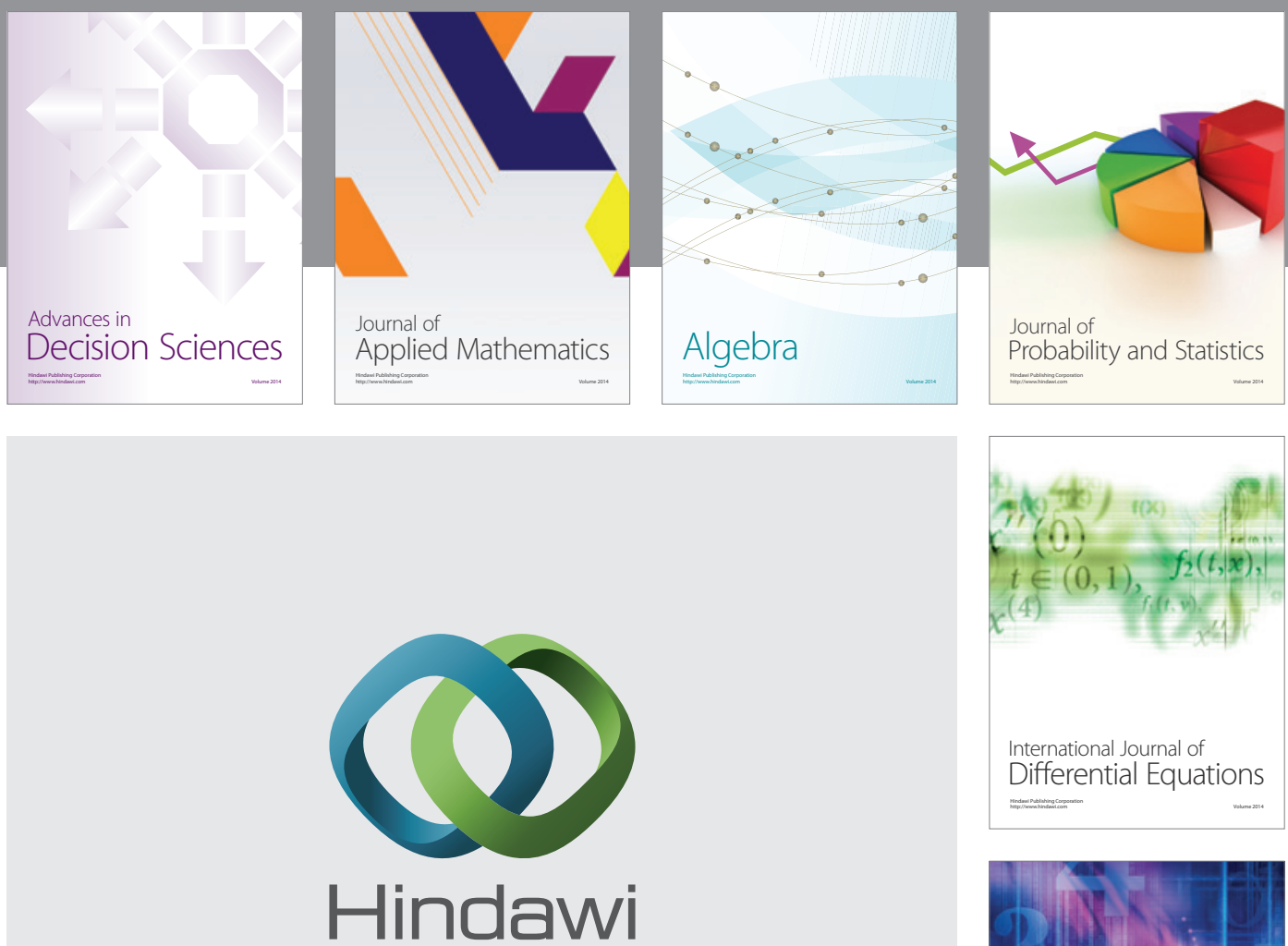

Submit your manuscripts at http://www.hindawi.com
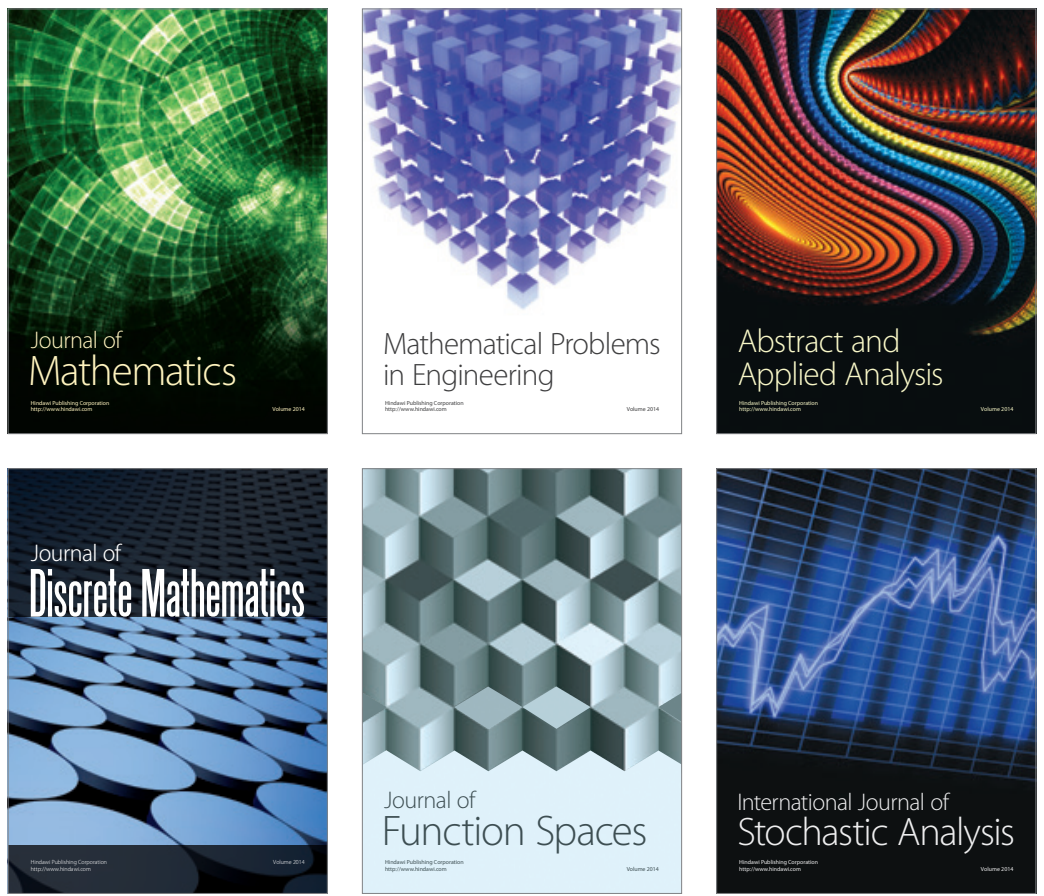

Journal of

Function Spaces

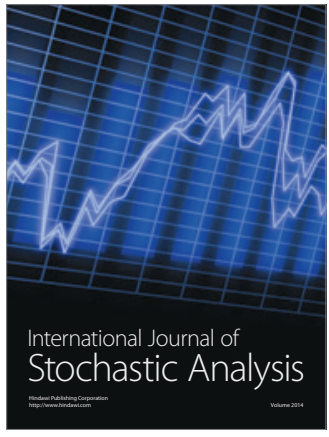

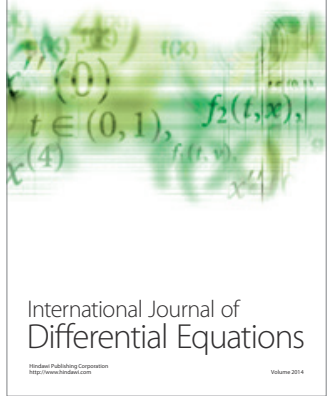
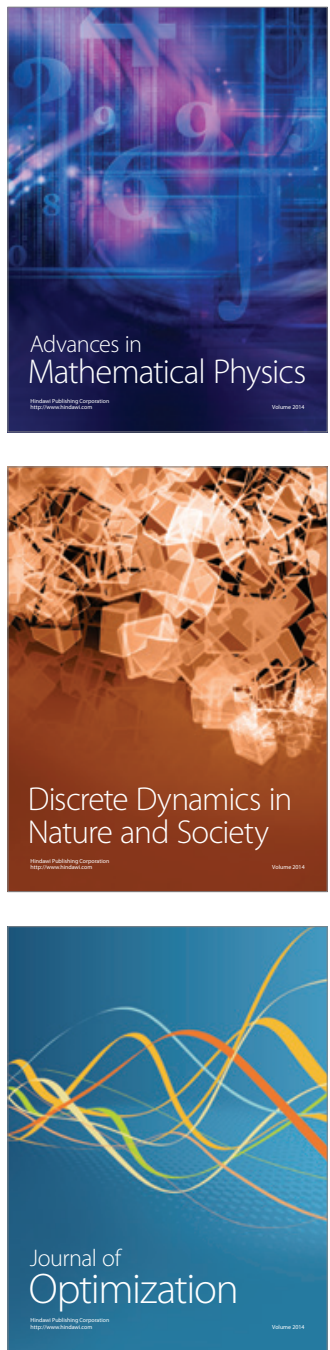